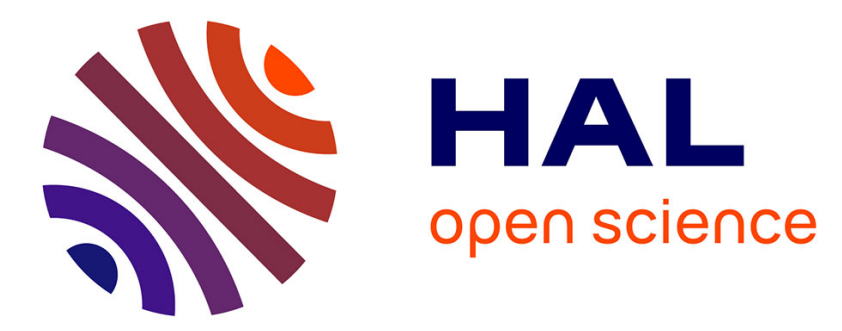

\title{
Renan jugé par la Congrégation de l'Index
}

Jean-Baptiste Amadieu

\section{To cite this version:}

Jean-Baptiste Amadieu. Renan jugé par la Congrégation de l'Index. Études Renaniennes, 2012, 113, p. $97-121$. hal-01326741

\section{HAL Id: hal-01326741 \\ https://hal.science/hal-01326741}

Submitted on 5 Jun 2016

HAL is a multi-disciplinary open access archive for the deposit and dissemination of scientific research documents, whether they are published or not. The documents may come from teaching and research institutions in France or abroad, or from public or private research centers.
L'archive ouverte pluridisciplinaire HAL, est destinée au dépôt et à la diffusion de documents scientifiques de niveau recherche, publiés ou non, émanant des établissements d'enseignement et de recherche français ou étrangers, des laboratoires publics ou privés. 


\section{Renan jugé par la Congrégation de l'Index}

Avec quatorze décrets de proscription, l'œuvre de Renan fut la plus mise à l'Index au $\mathrm{XIX}^{\mathrm{e}}$ siècle. La Congrégation romaine de l'Index inscrivit dans le catalogue de l'Index librorum prohibitorum successivement Le Livre de Job, Étude d'histoire religieuse, De l'origine du langage, Histoire générale et système comparé des langues sémitiques et Averroès et l'averrö̈sme en 1859, Le Cantique des cantiques en 1860, Vie de Jésus en 1863, Les Apôtres en 1866, Questions contemporaines et Saint Paul en 1869, Les Évangiles et la seconde génération chrétienne en 1877, L’Antéchrist et L'Église chrétienne en 1881, MarcAurèle et la fin du monde antique et L'Ecclésiaste en 1882, Nouvelles Études d'histoire religieuse en 1884, Histoire du peuple d'Israël en 1891, Souvenirs d'enfance et de jeunesse et Feuilles détachées faisant suite aux souvenirs d'enfance et de jeunesse en 1892, enfin Histoire du peuple d'Israël en 1894. Renan est l'auteur le plus examiné et le plus condamné par l'Index au XIX ${ }^{\mathrm{e}}$ siècle. À titre de comparaison, Comte, Cousin et Taine font chacun l'objet d'un seul décret de proscription; Quinet (trois décrets de l'Index) et Michelet (cinq décrets) font encore pâle figure face au palmarès de l'auteur de la Vie de Jésus. S'il est le plus proscrit en nombre de décrets, dans l'ordre qualitatif, en revanche, la censure est indigente. Contrairement à la condamnation de Lamennais explicitée par deux encycliques pontificales, à celle de Rosmini-Serbati notifiée par un décret du Saint-Office mentionnant les propositions incompatibles avec la foi de l'Église, ou encore à celle de Georg Hermes censuré par un bref de Grégoire XVI, ou plus tard à celles du modernisme, les décrets frappant l'œuvre de Renan ne s'accompagnent pas d'exposés des motifs de proscription. Avant l'ouverture des Archives de la Congrégation pour la Doctrine de la foi (ACDF) en 1998 par le cardinal Joseph Ratzinger, on ignorerait pour quelles raisons dix-neuf titres de Renan furent mis à l'Index par Rome, bien que l'on pût en deviner l'essentiel. Aucun acte du magistère ecclésiastique ne définit un corps de doctrine hétérodoxe que serait l'hérésie « renanienne ». Depuis l'ouverture des archives historiques de l'Index et de l'Inquisition (ouverture à laquelle rêvait Renan ${ }^{1}$ ), on sait le déroulement des procès en mise à l'Index; on connaît en particulier les noms des rapporteurs à qui la Congrégation a confié l'examen de chaque titre poursuivi, ainsi que le

\footnotetext{
${ }^{1}$ Il écrivait ainsi à la princesse Julie Bonaparte en 1865 : « Je ne crois pas [...] que la Bibliothèque du Vatican, en supposant que des savants libres puissent un jour y travailler à l'aise, réserve de grandes surprises en ce qui concerne l'antiquité. Ce sont les Archives pontificales et celles de l'Inquisition qui offriraient des trésors pour l'histoire du moyen âge et pour celle des temps modernes. » (Ernest Renan, CEuvres complètes, éd. Henriette Psichari, Paris, Calmann-Lévy, 1961, t. X : Correspondance 1845-1892, p. 423.)
} 
contenu de leur rapport. Ce rapport, nommé votum, éclairait les débats des consulteurs et des cardinaux de l'Index avant que ceux-ci décrètent la proscription.

\section{PROCEDURES CONTRE L'EEUVRE DE RENAN}

\section{A. Chronologie des décrets}

La Congrégation de l'Index poursuit avec une rare application l'œuvre de Renan. Cette constance est peu commune dans la mesure où le tribunal n'a pas les moyens humains de tout examiner et frappe symboliquement un ou quelques titres d'un auteur (par exemple NotreDame de Paris et Les Misérables de Hugo, le reste n'ayant pas fait l'objet d'un examen ; de même, de Comte, seul le Cours de philosophie positive est lu et mis à l'Index). Les quatorze décrets contre l'œuvre de Renan témoignent du danger que le Saint-Siège redoute pour ses fidèles. L'Index réagit assez vite aux publications de Renan, puisqu'à partir de 1860, onze des quinze titres mis à l'Index le sont l'année même de leur parution

B. Les quatre principaux censeurs de Renan

Si dix-neuf titres de Renan furent mis à l'Index, il n'y eut pourtant pas dix-neuf examinateurs de ses ouvrages. Certains de ses censeurs sont reconduits dans leur office. Ils partagent les mêmes domaines de spécialité avec Renan dont ils sont familiarisés avec l'œuvre. Sur les huit censeurs qui analysèrent ses livres, quatre ont examiné une quinzaine d'ouvrages de l'auteur. Ces principaux rapporteurs sont en quelque sorte les spécialistes de Renan au sein de la Congrégation et gardent la mémoire des procédures.

Le premier est Bernard Smith (1812-1892) ${ }^{2}$. Ce bénédictin irlandais est reçu docteur en philosophie (1834), puis en théologie (1840), avant d'entrer au Mont-Cassin en 1847. Deux ans plus tard, il est professeur de théologie et d'hébreu dans plusieurs établissements

\footnotetext{
${ }^{2}$ Voir Herman H. Schwedt, Tobias Lagatz, Prosopographie von römischer Inquisition und Indexkongregation, 1814-1917, Paderborn, Ferdinand Schöningh, série Grundlagenforschung: 1814-1917, coll. Römische Inquisition und Indexkongregation (dir. Hubert Wolf), 2005, vol. 2, 1390-1398.
} 
universitaires ecclésiastiques à Rome. Il entre en 1850 dans la Congrégation de l'Index comme consulteur. Avant les examens de Renan, le tribunal lui a confié divers rapports, en particulier sur les œuvres de Bailly (au moment de la crise «gallicane »), de John Stuart Mill, de Victor Cousin (Du vrai, du beau et du bien, examiné mais non mis à l'Index), des manuels de philosophie et de théologie. C'est probablement en raison de ses compétences en hébreu qu'on lui confie l'édition savante du Livre de Job par Renan en 1859, puis celle du Cantique des cantiques en 1860.

Le barnabite Carlo Giuseppe Vercellone (1814-1869) ${ }^{3}$ examine les Études d'histoire religieuse, De l'origine du langage, l'Histoire générale et système comparé des langues sémitiques en 1859, puis Les Apôtres en 1866. Comme Smith, Vercellone enseigne la théologie et la philosophie, notamment à Rome à partir de 1844. Il est membre de l'académie de religion catholique (1846) et assistant général de son ordre (1850). Il est nommé consulteur de l'Index en 1850. Quand il examine Renan, il a déjà rédigé des vota notamment sur Victor Cousin et le Dictionnaire de Bouillet. Il corrige en 1860 la traduction de la Bible par l'abbé Glaire. L'un de ses domaines de spécialité est l'Écriture sainte : il examine Les Évangiles. Examen critique et comparatif des trois premiers Évangiles de Gustave d'Eichthal, puis l'Histoire élémentaire et critique de Jésus d'Alphonse Peyrat.

Le troisième grand censeur de Renan est Giuseppe Pennacchi (1831-1913 ?) $)^{4}$. On sait peu de choses de ce prêtre séculier en dehors de sa carrière de curie. Ce professeur d'ecclésiologie entre dans la Congrégation de l'Index en 1868 et à l'académie de religion catholique en 1879. Canoniste et historien de l'Église, il a commis des expertises pour l'Index en Écriture sainte : les vota sur l'Esposizione critica della Genesi de Gioja et sur Le Procès de Jésus-Christ d'Aurélien Scholl. De Renan, il examine L'Antéchrist et L'Église chrétienne en 1881, Marc-Aurèle et L'Ecclésiaste en 1882 et les Nouvelles Études d'histoire religieuse en 1884.

Le quatrième examinateur principal est Luigi Tripepi (1836-1906) ${ }^{5}$. Il compose les vota sur les tomes I à III de l'Histoire du peuple d'Israël en 1891, les Souvenirs d'enfance et de jeunesse et les Feuilles détachées faisant suite aux souvenirs d'enfance et de jeunesse en 1892, enfin sur les tomes IV et V de l'Histoire du peuple d'Israël en 1894. Avec une large formation de juriste, d'historien, de théologien et de philosophe, Luigi Tripepi mène une brillante carrière de Curie, en même temps qu'il compose plus d'une trentaine d'ouvrages

\footnotetext{
${ }^{3}$ Ibid., p. 1539-1546.

${ }^{4}$ Ibid., p. 1153-1159.

${ }^{5}$ Ibid., p. 1498-1504.
} 
scientifiques, littéraires et théologiques. Il est élevé au cardinalat en 1901. Son entrée à l'Index comme consulteur date de 1878. En 1884, il est préfet de la commission pontificale des études historiques. C'est à lui que Léon XIII confie le soin d'ouvrir les Archives vaticanes, dont il est préfet à partir de 1892. Membre de l'académie de religion catholique depuis 1894, il la préside à partir de 1903. À la Congrégation de 1'Index, il se montre un censeur fin et minutieux. Son examen du roman de Zola Rome est la plus détaillée des censures littéraires pour tout le XIX ${ }^{\mathrm{e}}$ siècle : elle s'étend sur quarante pages imprimées et constitue un modèle du genre. Dans des domaines proches de ceux de Renan, il a rédigé les vota sur Le Christianisme et ses origines d'Ernest Havet et sur Les Erreurs scientifiques de la Bible d'Émile Ferrière.

\section{Déroulement des procédures}

Les normes réglementant la méthode à suivre dans l'examen des livres par l'Index sont fixées depuis la constitution apostolique Sollicita ac provida de Benoît XIV (1753). À la suite d'une plainte dénonçant une œuvre et explicitant les motifs d'incrimination, le secrétaire de la Congrégation de l'Index et le cardinal-préfet, s'ils jugent que la dénonciation est fondée, choisissent parmi les consulteurs un rapporteur pour rédiger un examen de l'œuvre, appelé votum ou censure. Après l'étude du propos incriminé, ce rapport se clôt sur un avis que le consulteur propose à la Congrégation : proscription, abandon des poursuites ou interdiction provisoire en attendant une version corrigée. $\mathrm{Au} \mathrm{XIX}^{\mathrm{e}}$ siècle, une fois le votum rédigé, il est imprimé et distribué aux membres de la Congrégation, aux consulteurs autant qu'aux cardinaux. Un premier jugement collégial, appelé congrégation préparatoire, réunit les consulteurs. Ils prononcent un avis consultatif sur la décision à prendre. Les cardinaux se réunissent ensuite en congrégation générale pour décréter sur l'œuvre. Seule leur voix est délibérative. Le décret cardinalice est ensuite soumis au pape qui le rend public en le promulguant.

Dans le cas de Renan, le modèle ordinaire des procédures est le suivant: le votum affirme l'incompatibilité des propositions de Renan avec la foi de l'Église et propose donc la proscription du livre incriminé ; la congrégation préparatoire des consulteurs vote dans le même sens en choisissant la proscription; la congrégation générale des cardinaux décrète également en conformité avec le votum et le scrutin des consulteurs; quant au pape, il n'oppose aucun obstacle au décret de proscription et ratifie ainsi la proscription. Il serait donc 
aussi inutile que fastidieux de décrire les quatorze procédures qui suivent ce schéma. Deux points particuliers méritent pourtant d'être signalés.

Le premier concerne les dénonciations. L'origine des dénonciations à l'Index est assez mystérieuse en général, puisque les archives de l'Index ne les conservent habituellement pas. Il arrive parfois qu'on puisse s'en faire une idée par des voies détournées. C'est en partie le cas pour Renan. Si l'on ne trouve pas dans les archives de l'Index la moindre trace de dénonciation, les archives de la Secrétairerie d'État conservent une correspondance entre la nonciature à Paris et la Secrétairerie au sujet de Renan à partir de l'été 1860.

Le 19 juillet 1860, le cardinal Giacomo Antonelli, secrétaire d'État de Pie IX, écrit au nonce à Paris ${ }^{6}$ pour lui demander un exemplaire du Cantique des cantiques par Renan afin de le transmettre à la Congrégation de l'Index pour qu'elle l'examine'. Carlo Sacconi lui envoie le volume le 11 août $^{8}$. La suite de la procédure est assez rapide. Bernard Smith, nommé rapporteur de l'œuvre, en rédige le votum. La congrégation préparatoire des consulteurs se réunit le 3 septembre; sept jours plus tard, les cardinaux réunis en congrégation générale décrètent l'interdiction de lecture du Cantique des cantiques par Renan. Le pape promulgue ensuite le décret du 10 septembre. Si cette correspondance entre la secrétairerie d'État et la nonciature éclaire en partie la phase liminaire de la procédure, elle ne permet pas de préciser avec certitude l'origine de la plainte. Cette dernière implique la plus haute autorité romaine après le pape, son secrétaire d'État, et passe par la voie diplomatique. La procédure est exceptionnellement rapide.

Une demande émanant de si haut a probablement conduit la nonciature à faire preuve d'une grande vigilance à l'égard de la production de Renan. Malgré le changement de nonce au début de l'automne 1861, le nouveau nonce Flavio Chigi (1810-1885) tient informée la secrétairerie d'État des activités du savant soupçonné d'hétérodoxie. Le 2 décembre 1861, Chigi écrit au secrétaire d'État au sujet de la chaire d'hébreu du Collège de France, et d'une autre en linguistique sémitique ou comparée, laquelle serait attribuée à Renan :

Celui-ci a beaucoup d'esprit, d'imagination et il écrit bien ; mais il ne possède pas une grande science et il fait montre d'impiété et d'incrédulité. Ses écrits tendent à détruire la Révélation, les Saintes Écritures et les fondements de la religion chrétienne. Il semblerait incroyable que le Gouvernement pensât lui donner une chaire, d'où seront attaquées chaque jour la Sainte Écriture et la Religion. Je sais

\footnotetext{
${ }^{6}$ Le nonce apostolique à Paris est alors Carlo Sacconi (1808-1889), jusqu'à son élévation au cardinalat en septembre 1861 .

${ }_{7}$ Archivio Segreto Vaticano (ASV), Archivio della Nunziatura Apostolica in Parigi, busta 160, f. 33r.

${ }^{8}$ ASV, Segreteria di Stato, Anno 1860, Rubrica 248, fasc. unico, f. 171r.
} 
que le cardinal-archevêque s'en est montré assez préoccupé, et qu'il en a parlé sérieusement au Ministre des cultes ${ }^{9}$.

Le 12 janvier 1862, le nonce confirme à la secrétairerie que Renan a été nommé à la chaire de langues hébraïque, chaldaïque et syriaque du Collège de France. Le ministre des cultes a tâché de rassurer le nonce en lui précisant que le nouveau professeur s'occupera seulement de recherches littéraires et philosophiques, et qu'il observera la réserve et le respect qui s’imposent face au caractère sacré de la Bible. Le nonce reste néanmoins perplexe : «Sperare della riserva, e del rispetto da un Professore che fa professione d'incredulità, e che nè suoi scritti attacca, e cerca distruggere la divina ispirazioni de' libri santi: è sperare l'impossibile ${ }^{10}$ !».

Moins de deux mois plus tard, le nonce écrit au cardinal Antonelli une relation du cours au Collège de France du 22 février 1862, au sujet duquel il retient une profession de foi panthéiste et la négation de la divinité de Jésus. L'impiété fut telle que le gouvernement luimême a dû suspendre l'enseignement, relate-t-il. Le cours s'est accompagné de scènes de désordres. La lettre au secrétaire d'État éreinte surtout la responsabilité du Ministre des cultes, qui n'ignorait pas la nature du personnage ${ }^{11}$.

Par la suite, les relations de la nonciature avec la secrétairerie ou la Congrégation de l'Index deviennent plus lâches. Le 27 juin 1863, le nonce Chigi envoie à la secrétairerie d'État un exemplaire de la Vie de Jésus, à transmettre à la Congrégation de 1'Index ${ }^{12}$. Et en 1877, le secrétaire d'État envoie à l'Index, au nom du nonce à Paris, Les Évangiles de Renan «pour l'usage qui conviendrait $»^{13}$.

Ce sont là les seuls indices sur la dénonciation, ou plus généralement sur la genèse, des procédures relatives à l'œuvre de Renan. La nonciature à Paris y occupe une place de premier plan. Au plus haut niveau de l'Église, on craint de voir les théories de l'auteur de la Vie de Jésus se répandre chez les fidèles.

\footnotetext{
${ }^{9}$ Traduction de ASV, Segreteria di Stato, Anno 1862, Rubrica 248, fasc. 1, f. 132v-133r. La cardinal-archevêque désigne ici l'archevêque de Paris, François-Nicolas Morlot (1795-1862), successeur de Mgr Sibour à la tête du diocèse depuis 1857 et grand aumônier de l'Empereur. Le ministre de l'instruction publique et des cultes est Gustave Rouland ; il occupe ce poste de 1856 à 1863.

${ }^{10}$ ASV, Segreteria di Stato, Anno 1862, Rubrica 248, fasc. 1, f. 135rv. Trad. : «Espérer de la réserve et du respect de la part d'un professeur qui fait profession d'incrédulité, et qui, dans ses écrits, attaque et cherche à détruire l'inspiration divine des livres saints, c'est espérer l'impossible ! »

${ }^{11}$ Lettre du $1^{\text {er }}$ mars 1862, ASV, Segreteria di Stato, Anno 1862, Rubrica 248, fasc. 1, f. 137r-138r.

${ }^{12}$ ASV, Segreteria di Stato, Anno 1863, Rubrica 248, fasc. 2, f. 45rv.

${ }^{13}$ Archivio della Congregazione per la Dottrina della fede (Archives de la Congrégation pour la doctrine de la foi, Palazzo del Santo Officio, 00120 Città del Vaticano. En abrégé : ACDF), Index, Atti e documenti, 1875$1877, \mathrm{n}^{\circ} 442$.
} 
La seconde remarque relative au déroulement des procédures concerne le débat sur l'extension des clausules de condamnation. La clausule désigne la partie de l'œuvre que la Congrégation de l'Index proscrit. Elles sont généralement nominatives : un ou plusieurs ouvrages sont interdits nommément. Parfois, la Congrégation condamne sous clausule générale : en 1895, le tribunal met ainsi à l'Index les opera omnia (toutes les œuvres) de Zola, sans mention particulière de titres ; en 1863, sont proscrits les omnia scripta romanensia (tous les écrits romanesques) des Dumas. Pour la période contemporaine, l'usage réserve plutôt les clausules générales aux œuvres de fiction. La production de Renan fait l'objet d'examens trop détaillés et réguliers pour tomber sous le coup d'une grande condamnation générale rétrospective. Le modèle usuel est nominatif : le votum traite d'un ou de plusieurs titres, qui sont jugés en congrégation préparatoire, puis condamnés nommément par les cardinaux. Les jugements collégiaux observent le processus nominatif sans le remettre en cause. À ce modèle constant, dérogent deux cas exceptionnels :

1. une proposition de restriction de la clausule à certains chapitres. En 1869, le rapporteur Guglielmo Andrea Audisio, chargé du votum sur les Questions contemporaines, argue que les cinq premiers chapitres ne traitent pas de questions relevant de la censure ecclésiastique (instruction supérieure en France, Institut de France, professeurs au Collège de France et chaires), mais de questions académiques sans rapport direct avec la doctrine de la foi ou les règles des mœurs. Aussi son rapport se conclut-il sur deux propositions de censure possibles qu'il soumet à l'avis des membres de la Congrégation :

- soit condamner seulement les dissertations incompatibles avec l'enseignement magistériel. Ce mode de censure, privilégié par le rapporteur, lui semble «plus raisonnable et plus opportun en ce temps où l'on crie contre les immenses proscriptions de l'Index et où des catholiques allemands signent des pétitions aux évêques pour demander l'abrogation de l'Index au prochain concile ${ }^{14}$;

- soit condamner le livre nommément, sans distinction de chapitres. Ce mode, qui est d'usage dans la Congrégation, lui paraît «plus expéditif»; le censeur ajoute que toutes les dissertations étant imprimées en un seul volume, il est impossible de séparer la partie du tout.

\footnotetext{
${ }^{14}$ Trad. de ACDF, Index, Protocolli 1865-1869, n 187, p. 3.
} 
L'Index suit le modèle usuel de la condamnation nominative, en ne précisant pas les chapitres condamnés après la mention des Questions contemporaines dans le décret du 12 juillet 1869 .

2. une proposition d'élargissement aux opera omnia. En 1894, à l'occasion de la dernière procédure, Luigi Tripepi examine les tomes IV et V de l'Histoire du peuple d'Israël, qui ont donné lieu à une publication posthume l'année précédente. La mort de Renan signifie la clôture de son corpus éditorial. Le censeur propose alors une condamnation opera omnia : «il me semble évident qu'aucun auteur de notre temps ne fut, plus que Renan, digne que toutes ses œuvres fussent mises à 1 'Index $»^{15}$. La proposition de Tripepi est débattue à la congrégation préparatoire du $1^{\mathrm{er}}$ juin 1894 . Le rapport de cette consultation collégiale est succinct : tous furent de l'avis du consulteur (selon la formule usuelle omnes fuerunt in voto consultoris). Le rapport ajoute cependant que toutes les œuvres doivent être proscrites :

- après un examen selon onze consulteurs ;

- $\quad$ sans examen selon deux d'entre eux.

Les cardinaux ne suivent pas les recommandations du rapporteur ni des consulteurs. Leur décret proscrit nommément les deux volumes, et aucune nouvelle procédure n'est ouverte pour juger l'ensemble des œuvres de Renan.

Malgré les propositions inverses de restriction ou d'élargissement de la clausule, les cardinaux s'en tiennent à la procédure nominative, conformément à la coutume dans le cas d'œuvres portant sur des sujets propres aux disciplines ecclésiastiques. Renan ne subit pas le même traitement que plusieurs dramaturges ou romanciers, pour lesquels l'œuvre fut condamnée en bloc : Sue, Dumas, Sand, Balzac, Stendhal, Champfleury, Murger, Zola. La régularité de ses examens et le refus de se servir de la clausule générale, en dépit de la lassitude de certains censeurs, mettent le professeur de langue sémitique à rang égal avec ces ecclésiastiques marginaux à l'égard desquels le tribunal évite le régime plus sommaire dont on use par exemple à l'encontre des écrits de fiction. L'érudition des ouvrages de Renan et leur contenu religieux, malgré l'indignation que suscitent ses conclusions dans le haut clergé romain, classent l'œuvre poursuivie parmi les traités savants touchant aux disciplines sacrées, envers lesquels la science ecclésiastique témoigne de son attention tout en s'exerçant à controverse.

\footnotetext{
${ }^{15}$ Trad. de ACDF, Index, Protocolli 1894-1896, n²8, p. 43.
} 


\section{CONTENU DES CENSURES ROMAINES}

\section{A. Quelques considérations nuancées}

Les textes de censure suivent assez rarement une structure balancée plaidoyerréquisitoire. Ils ont plutôt pour finalité de signaler si le texte déféré devant le tribunal s'écarte des propositions de foi, et le cas échéant dans quels passages de l'œuvre et avec quel degré d'écart. Dans leur ensemble, les vota sur Renan ne dérogent pas à cet usage. Pourtant des considérations plus nuancées atténuent parfois la sévérité des censures.

Les nuances portent d'abord sur l'attitude encore incertaine de Renan. Les premières censures n'excluent pas la possibilité d'un auteur qui deviendrait un savant catholique, voire un champion de l'Église. Dans son votum d'avril 1859 sur Le Livre de Job, le censeur Bernard Smith, qui dit d'ailleurs connaître personnellement l'auteur, pense que Renan n'est pas nécessairement mal intentionné, que ce disciple s'est plutôt laissé abusé qu'il n'est un mauvais maître ; il eût pu être un «serviteur de la vérité et de l'Église » :

Certamente il Sig. Renan è giovane di gran talento: e se avesse lasciato quella scuola Razionalistica panteistica, che oggi fa tanto progresso nella Francia, servirebbe la causa della verità e della Chiesa. Io lo conosco personalmente. $\mathrm{Ma}$ quando lo conobbi, non era ancora divenuto Ateo per la sua amicizia con V. Cousin ${ }^{16}$.

Ce point de vue change au cours des procédures suivantes, au moins depuis la Vie de Jésus selon Vercellone ${ }^{17}$. Dans son votum sur Les Évangiles, le jésuite Angelini Rota tient en 1877 des propos sur Renan qui ne laissent plus de doute sur la figure auctoriale, désormais constituée et condamnée à une infamie perpétuelle, de sorte que les œuvres de Renan sont a priori suspectes :

Il nome del Renan condannato a perpetua infamia nella memoria de'posteri è un grave argomento per disapprovare le opere uscite dallo stesso autore. E al fermo in questa nuova opera [Les Évangiles] egli segue la medesima via in che s'era messo scrivendo la vita di G. Cristo ${ }^{18}$.

\footnotetext{
${ }_{16}^{16}$ ACDF, Index, Protocolli 1858-1861, n³5, p. 8.

17 «Poche parole basteranno per dare una sufficiente notizia di questo libro [Les Apôtres] a chi deve giudicarlo. L'Autore proseguendo la via sulla quale si era messo col suo troppo famoso libro Vie de Jésus, continua a impugnare tutto ciò che v'ha di sovrannaturale nell'origine storica del cristianesimo. »(ACDF, Index, Protocolli 1865-1869, n83, p. 1.)

${ }^{18}$ ACDF, Index, Protocolli 1875-1878, n³26/IV, p. 1.
} 
La Vie de Jésus constitue un tournant dans l'élaboration de l'identité hétérodoxe de Renan. Curieusement, les diplomates de l'Église semblent plus précocement méfiants à son égard que les censeurs de l'Index.

La deuxième concession que les censeurs accordent à l'œuvre de Renan concerne son érudition. Le nonce Chigi confiait au secrétaire d'État à son sujet : «scrive bene ; ma non possede molta scienza ${ }^{19}$. Ce jugement ne reflète pas la pensée des censeurs, qui, au demeurant, peuvent rendre hommage à l'érudition de Renan. En 1859, dans son votum sur Averroès et l'averroüsme, Semenenko, même s'il relève plusieurs propositions du livre dignes de condamnation, qualifie la science de Renan de «non mediocrem $»^{20}$. Dans le jugement de Smith cité précédemment, celui-ci lui reconnaissait un grand talent.

La troisième nuance apportée à la sévérité des censures est la qualité du style de Renan, que reconnaissait déjà le nonce Chigi. Tripepi, qui examine en 1891 l'Histoire du peuple d'Israël, note que cet ouvrage est pire encore que les précédents qui furent mis à l'Index. Il reconnaît la réussite littéraire de l'ouvrage :

Ed inoltre alcune attrattive di stile e di descrizioni che al Renan non si possono negare, nonchè l'apparato di fallace erudizione e di continue citazioni per recenti studi orientali e biblici, e la forma di apparente convincimento e di calmo ragionamento che si ostenta, rendono i tre volumi assai più pericolosi, segnatamente pe'giovani ${ }^{21}$.

L'ouvrage est plus dangereux que les précédents qui furent mis à l'Index, en partie en raison des attraits stylistiques et rhétoriques, qui, par l'apparence de sérieux et d'impartialité, renforcent sa séduction, son crédit et donc son péril sur les lecteurs les plus vulnérables. L'année suivante, au sujet des Souvenirs d'enfance et de jeunesse suivis des Feuilles détachées faisant suite aux souvenirs d'enfance et de jeunesse, le même censeur fait des éloges de l'écriture aussi prononcés et ambigus :

La forma di narrazione o di romanzo, che domina nei due libri, e la maniera di scrivere spigliata, immaginosa, spesso poetica, gonfia di sentimentalismo e, al certo, in una lingua elegante, sono attrattive, che inducono, specialmente $\mathrm{i}$ giovani, a leggere, ad affezionarsi all'autore, alle sue follie, ai suoi errori, alle sur impudenze, alle sue apostasie, ed a quello smanceroso parossismo che si manifesta in tutte le pagine, e che, chiaramente, è la conseguenza di una vita non solo molle, ma ancora forse effeminata, voluttuosa, dissoluta ${ }^{22}$.

\footnotetext{
${ }^{19}$ ASV, Segreteria di Stato, Anno 1862, Rubrica 248, fasc. 1, f. 132v-133r.

${ }^{20}$ ACDF, Index, Protocolli 1858-1861, n47, p. 1.

${ }^{21}$ ACDF, Index, Protocolli 1889-1891, n 133, p. 1.

${ }^{22}$ ACDF, Index, Protocolli 1891-1894, n56, p. 1-2.
} 
Plus encore que dans la censure de l'Histoire du peuple d'Israël, l'apparente apologie du style de Renan tourne à une plus grande condamnation. Tripepi suit les étapes d'une rhétorique formelle dont de nombreuses censures d'œuvres littéraires sont imprégnées :

1. l'œuvre est mauvaise dans son contenu intellectuel, lequel s'écarte de l'enseignement dogmatique et moral de l'Église ;

2. l'œuvre est séduisante en raison des qualités littéraires de son écriture ;

3. l'œuvre est donc dangereuse pour un public vulnérable et facilement dupe du fait de la combinaison de la malice et du danger.

Le constat des mérites littéraires est trop à double tranchant pour constituer à proprement parler une nuance dans les vota qui équilibrerait la sévérité censoriale. Dans l'ensemble, les nuances sont anecdotiques à l'intérieur des rapports de l'Index. Quand elles ne concernent pas un espoir potentiel au début de la carrière intellectuelle de Renan quant à une possible conversion, les qualités reconnues à l'auteur ne le sont que de façon soit marginale (érudition) soit équivoque (style). La plus grande part des vota restent consacrée aux griefs de l'Église contre les œuvres poursuivies.

\section{B. Principaux griefs}

\section{Philologie, langage et interprétation de la Bible}

Dès la première censure, l'Index s'en prend aux travaux d'érudition que mena Renan sur la Bible. Smith lui reproche de reprendre la distinction usuelle de l'exégèse rationaliste entre un rédacteur élohiste et un rédacteur jéhoviste :

Per vedere che i due nomi Elohim e Jeoha non provano diversi scritti ed autori diversi, basta osservare che si trovano nello stesso capitolo, nei versi stessi, e nelle medesime frasi « Istae sunt generationes coeli et terrae, quando creata sunt in die, quo fecit Jehovah - Elohim coelum et terram. » Gen. 11, 4. Dipiù nella Genesi le parole Jehovah, ed Elohim sono usate indistintamente.

Il Signor Renan fa torto alla Germania quando dice che tutti i suoi dotti critici difendono questa ipotesi, perchè fra i cattolici non trova nessun difensore, $\mathrm{e}$ fra gli stessi razionalisti moltissimi non l'ammettono ${ }^{23}$.

Smith conteste encore les analyses de Renan sur plusieurs points d'exégèse : lorsque le commentateur du Livre de Job affirme que le scheol des Hébreux, où l'on descendait après la mort, « est souvent difficile de discerner du tombeau », le censeur réplique : «Questo è falso. I Giudei esprimono letteralmente il sepolcro col nome Heber ed il sito delle anime dopo la

\footnotetext{
${ }^{23}$ ACDF, Index, Protocolli 1858-1861, n³5, p. 4.
} 
morte per i nomi scheol e avaddon, l'Orco dei Latini ${ }^{24}$. » En ce qui concerne les erreurs de traduction, Smith défend la justesse de la traduction de S. Jérôme et montre la cohérence de la Vulgate avec la version originale dont se réclame Renan.

La deuxième censure de Smith porte également sur les analyses critiques du texte biblique par Renan, à l'occasion de la publication du Cantique des cantiques. Le votum de 1860 ne porte plus sur des questions de traduction, mais sur deux points :

1. la canonicité du livre : le censeur s'appuie sur l'autorité des décrets de l'Église (en particulier la condamnation des thèses de Théodore de Mopsueste et les décrets du concile de Trente), de la Synagogue, du Midrash et de la Torah. L'argument d'autorité pourrait fausser le « débat », si débat il y avait et que la controverse était publique et non pas interne à un tribunal de la communauté ecclésiale ;

2. l'objet et le but de l'auteur du livre saint. La démonstration s'appuie également sur un argument d'autorité : «Le Parafrasi Caldaiche, Jarchi, e tutti i più dotti Rabini convengono che il Poema sia spirituale, o mistico ${ }^{25}$. Les Pères et les auteurs, d'Origène à S. Bernard, le protestant Horne lui-même, adoptent la thèse d'une lecture spirituelle. Cette généalogie, ajoute Smith, est plus ancienne que celle des partisans du sens littéral, laquelle remonte à Théodore de Mopsueste, puis se développe avec Sébastien Castellion, Grotius et Jean Le Clerc. Mais «Il N[ostro] A[utore] però non segue tale opinione, che condanna la Cantica come un libro immorale, o di influenza cattiva od oscena ${ }^{26}$. " La thèse de Renan selon laquelle l'épouse de Salomon avait un amant paraît inacceptable au censeur. Son commentaire sur le livre sacré «è infetto del vizio dominante del Razionalismo biblico», et «merita di esser proibito $»^{27}$.

C'est une constante des censures de Renan que de contester les traductions et les commentaires qu'il propose. En 1882, le rapporteur Pennachi, dans son examen de L'Ecclésiaste, doute de la justesse de la traduction. Elle lui semble forcée. Renan oriente le texte dans le sens de sa thèse, selon laquelle l'auteur est sceptique : «Nè migliore è la traduzione francese che Renan ci dà nella seconda parte del suo libro ; tutto ivi cospira a dimostrare l'asunto, essere cioè l'autore dell'Ecclesiaste uno scettico ${ }^{28}$. »

Quand la censure s'applique à l'examen de la philologie biblique, les développements se spécialisent soit sur le langage, soit sur le contenu biblique et son interprétation. Le votum sur

\footnotetext{
${ }^{24}$ Ibid., p. 5.

${ }^{25}$ ACDF, Index, Protocolli 1858-1861, nº79, p. 4-5.

${ }^{26}$ Ibid., p. 5.

${ }^{27}$ Ibid., p. 6.

${ }^{28}$ ACDF, Index, Protocolli 1882-1884, n³2, p. 2.
} 
l'Étude d'histoire religieuse et De l'origine du langage composé par Vercellone illustre le premier type de développements. Le ton est autant censorial qu'ironique. La préface, au dire du censeur, est «un vero capolavoro di empietà e di ridicolezza $»^{29}$. Vercellone apprécie l'analyse de l'origine du langage. Il résume d'abord la théorie du langage de Renan en cinq principes, appuyés de citations prises en divers endroits de l'ouvrage :

1. Le langage humain n'a pas d'origine surnaturelle ;

2. Le langage est le produit de l'activité spontanée de l'homme ;

3. La liaison du sens et du mot n'est jamais arbitraire, elle est toujours motivée ;

4. Les langues appartiennent à de grandes familles; elles peuvent se modifier selon le climat, la politique, les événements intellectuels et religieux, mais ne sortent jamais du type général auquel elles appartiennent ;

5. Les langues-mères sont nombreuses.

Ces cinq propositions ne forment pas un corps de doctrine nécessairement faux, selon l'interprétation qu'on leur donne : "potrebbe forse intendersi sanamente, ma il Renan nello svolgerla vi va innestando pressoche tutti gli errori più principali del razionalismo panteistico, di cui si mostra evidentemente seguace $»^{30}$. Les interprétations « rationalistes panthéistes » hétérodoxes concernent, outre la négation d'une action surnaturelle de Dieu dans le monde, des affirmations anthropologiques incompatibles avec la foi de l'Église et indifférentistes :

1. «Il Renan mette in dubbio l'unità della specie umana ${ }^{31}$. »

2. «Il Renan attribuisce all'uomo primitivo un'origine autoctona $»^{32}$, au sens de lois naturelles qui auraient déterminé la formation du monde et de l'homme plutôt que de l'évolution ;

3. «Il Renan si attiene alla sentenza dei bibliologi razionalisti riguardo all'origine e alla natura dei racconti biblici ${ }^{33}$.»

4. «Il Renan considera le tre religioni, giudaica, cristiana e musulmana come sostanzialmente identiche ${ }^{34}$. »

5. «Il Renan nega la possibilità e conseguentemente l'esistenza del miracolo, la libertà di Dio e la sua Provvidenza ${ }^{35}$.»

\footnotetext{
${ }^{29}$ ACDF, Index, Protocolli 1858-1861, n48, p. 1.

${ }^{30}$ Ibid., p. 7.

${ }^{31}$ Ibid., p. 8.

${ }^{32}$ Ibid.

${ }^{33}$ Ibid., p. 9.

${ }^{34}$ Ibid., p. 10.

${ }^{35}$ Ibid.
} 
La censure de l'Histoire générale et système comparé des langues sémitiques qui clôt le votum de Vercellone prolonge la première considération censoriale de l'analyse précédente, notamment la distinction qu'établit Renan entre la race sémitique et la race indo-européenne, dont voici un extrait :

Adunque la razza semitica rappresenta realmente une combinaison inférieure de la nature humaine. Essa non ha quell'altezza di spiritualismo che l'India e la Germania sole hanno conosciuto. La coscienza semitica è chiara, ma poco estesa ; ella comprende bene l'unità, ma non la moltiplicità. Questa è la gloria della razza semitica d'aver avuto la nozione della divinità e d'averla propagata agli altri popoli. Ma d'onde l'hanno avuta questa nozione? L'avaient trouvé dans les instincts les plus impérieux de leur esprit et de leur cœur; la qual cosa era impossibile agli altri popoli ${ }^{36}$

Le censeur voit dans la lecture des Écritures par Renan une méthode qui ne se contente pas de naturaliser le texte biblique. Cela suffirait à justifier une mise à l'Index. La lecture renanienne fait aussi de la Bible l'expression d'une culture déterminée, qui plus est d'une culture défaillante, ôtant ainsi à l'Écriture non seulement son caractère surnaturel mais sa prééminence sur tout autre livre.

Parmi les autres types de censure relatifs à l'interprétation biblique, les relevés des incohérences du texte sacré sont rapportés par les examinateurs de l'Index comme autant de lectures sacrilèges. Si certaines censures se dispensent de qualifier l'erreur tant sa seule citation paraît accablante, les propositions sur l'incohérence de la Bible sont régulièrement réfutées, tel cet extrait de la censure des Évangiles et la seconde génération chrétienne par Angelini Rota au sujet des variations des textes évangéliques concernant les larrons crucifiés en même temps que Jésus :

Pag. 265. « Il y a de sa part [l'évangéliste Luc] préoccupation, parti pris, idée fixe. Son coup le plus hardi a été de convertir un des larrons du Calvaire. Selon Marc et Matthieu, les deux malfaiteurs insultaient Jésus. Luc prête à l'un d'eux un bon sentiment : « Nous, nous l'avons mérité : mais ce juste !... »

E qui mi si permetta osservare con S. Girolamo, s. Agostinop, e Ambrogio, che Marco e Matteo dissero per sillepsi in plurale ciò che è singolare, secondo che in più luoghi usa la S. Scrittura : all'incontro i Padri Greci, Origene, il Crisostomo, Teofilatto, Atanasio stimano, che da prima amendue i ladroni bestemmiassero G. C. e che poco appresso uno di essi si convertisse ${ }^{37}$.

Cet extrait illustre d'ailleurs l'un des reproches régulièrement adressé par l'apologétique ecclésiastique aux lectures «rationalistes» de la Bible : la lecture exclusive du texte en faisant abstraction de sa tradition interprétative.

\footnotetext{
${ }^{36}$ Ibid., p. 12.

${ }^{37}$ ACDF, Index, Protocolli 1875-1878, n³26/IV, p. 3.
} 
Mais en matière d'interprétation biblique, le rationalisme que poursuivent les censeurs romains consiste avant tout à nier les interventions surnaturelles dans l'histoire sainte : «Posta questa base, l'Autore comincia dall'attribuire ad una illusione il prodigio dalla risurrezione di Cristo ; et poscia spiega i miracoli operati dagli Apostoli come invenzioni o imposture dei primi tempi $»^{38}$ remarque ainsi Vercellone dans son votum sur Les Apôtres. Le postulat rationaliste conduit nécessairement à une critique de certaines croyances ecclésiastiques, parmi lesquelles l'article du credo sur la résurrection de Jésus-Christ, en évoquant des motifs plus ou moins injurieux pour expliquer ces croyances (illusion ou imposture). La notion de rationalisme imprègne les censures romaines de Renan.

\section{Le rationalisme}

Le rationalisme est en effet le leitmotiv des censures de Renan, de 1859 à 1894. Dans son encyclique Qui Puribus de 1846, Pie XI condamne le rationalisme, selon lequel «les mystères très saints de notre religion sont des imaginations et des inventions des hommes ». Le pape y définit un ordonnancement orthodoxe entre foi et raison :

la raison humaine doit s'enquérir diligemment sur le fait de la Révélation, pour savoir avec certitude que Dieu a parlé et pour lui rendre, comme l'enseigne très sagement l'Apôtre, « un hommage conforme à la raison». Qui donc ignore ou peut ignorer qu'il faut avoir une confiance totale en Dieu quand il parle, et que rien n'est plus conforme à la raison elle-même que d'acquiescer à ce qu'elle aura reconnu comme révélé par Dieu, qui ne peut ni se tromper ni nous tromper?

Le Syllabus de 1864 condamne plusieurs propositions qualifiées de rationalistes, parmi lesquelles : «Les prophéties et les miracles exposés et racontés dans les Écritures saintes sont des fables des prêtres, et les mystères de la foi chrétienne sont le fruit d'inventions philosophiques; et dans les livres des deux Testaments sont contenues des inventions mythiques; et Jésus Christ lui-même est une fiction mystique » (septième proposition condamnée). Six ans plus tard, lors du concile du Vatican, la constitution dogmatique Dei Filius anathématise les lectures rationalistes de la Bible: «Si quelqu'un ne reçoit pas les livres de la sainte Écriture comme sacrés et canoniques, dans leur intégrité et avec toutes les parties, tels qu'ils sont énumérés par le saint concile de Trente, ou s'il nie qu'ils soient divinement inspirés, qu'il soit anathème ».

Les censures de Renan sont contemporaines de ces condamnations du rationalisme. De façon récurrente, les rapporteurs dénoncent l'usage de la seule raison, séparée de la foi et

\footnotetext{
${ }^{38}$ ACDF, Index, Protocolli 1865-1869, nº3, p. 2.
} 
récusant les facteurs surnaturels ou la tradition ecclésiastique. Rationaliste est souvent associé à panthéiste sous leur plume, dans la mesure où le rationalisme en vient à n'attribuer à la vie d'autre origine ni explication que le monde, lequel acquiert ainsi les attributs divins (éternité, non-création, causalité, finalité, etc.) Les propositions rationalistes notées par les censeurs nient la création du monde par Dieu, l'origine surnaturelle du langage, l'inspiration divine de la Bible, la possibilité des miracles, la nature divine du Christ, le sens providentiel de l'Histoire $^{39}$, etc.

La censure de 1891 sur l'Histoire du peuple d'Israël, due à Luigi Tripepi, est emblématique de cette dénonciation du rationalisme associé au panthéisme. Le rapporteur note d'abord que cet ouvrage est pire que les précédents qui furent mis à l'Index. L'érudition et la modération de ton qu'affecte Renan apportent au livre une capacité de séduction qui, conformément à la rhétorique jurisprudentielle usuelle du tribunal, le rend d'autant plus dangereux :

Ed inoltre alcune attrattive di stile e di descrizioni che al Renan non si possono negare, nonchè l'apparato di fallace erudizione e di continue citazioni per recenti studi orientali e biblici, e la forma di apparente convincimento e di calmo ragionamento che si ostenta, rendono $\mathrm{i}$ tre volumi assai più pericolosi, segnatamente pe'giovani ${ }^{40}$.

Il est inutile de s'appesantir sur ce lieu, déjà abordé plus haut. L'intérêt de la censure de l'Histoire du peuple d'Israël, au regard de la question du rationalisme, est le résumé sous forme de propositions que Tripepi soumet à ses confrères et aux cardinaux. Après la censure de plusieurs citations de Renan, et au milieu de la censure, il récapitule les principales caractéristiques de l'hétérodoxie de l'auteur :

Dalle cose accenate si vede che, tra altro, Renan $1^{\circ}$ non ammette l'esistenza di un vero Dio ; $2^{\circ}$ riconosce la materia eterna ed il suo evoluzionismo alla maniera di Darwin ; $3^{\circ}$ pensa che il racconto Biblico è dedotto da quello di popoli idolatri, ma rivestito a forma monoteista del nomade semita $; 4^{\circ}$ crede la Bibbia un tessuto di favole, mostruosità, non differenti, nella sostanza, da quelle del politeismo, ma solo rese apparamente più credibili dalle tinte monoteistiche $; 5^{\circ}$ afferma che nella Bibbia comme nelle teorie babilonesi da cui ha attinto, vi è, in fondo, ammesso l'evoluzionismo darwiniano ${ }^{41}$.

Mise à part la première proposition, qui relève du rationalisme en général, les autres propositions définissent l'hétérodoxie renanienne comme un composé d'interprétation rationaliste de la Bible et de panthéisme, ici non nommé mais signifié par la croyance en

\footnotetext{
${ }^{39}$ Pour l'anecdote, dans sa censure de Marc-Aurèle, Pennachi reproche à Renan de reprendre la thèse de Gibbon attribuant la décadence de Rome au christianisme. Voir ACDF, Index, Protocolli 1882-1884, n³ 3, p. 2.

${ }^{40}$ ACDF, Index, Protocolli 1889-1891, n ${ }^{\circ} 133$, p. 1.

${ }^{41}$ Ibid., p. 11.
} 
l'éternité de la matière et en son évolution. Les deux mentions de l'évolutionnisme font explicitement référence à Darwin, dont Renan confiait avoir entrevu plusieurs résultats dès ses années de séminaires ${ }^{42}$. La négation de l'intervention divine dans l'histoire du monde et son remplacement par l'idée d'évolution interne à la matière et au vivant comme facteur explicatif du développement de la vie constituent les aspects essentiels de son hétérodoxie. En matière d'interprétation biblique, les propositions 3 et 4 suivent une formulation proche des énoncés condamnés par le Syllabus (caractère fabuleux d'épisodes bibliques) et Dei Filius (inspiration divine de la Bible).

Avant de conclure sa censure, Tripepi propose un nouveau résumé, qui ajoute au précédent l'idée d'une sorte de pente fatale :

l'opera del Renan è un tessuto delle teoriche più aperte ed assurde del naturalismo moderno; si occupa di proposito ad escludere dalla storia e dalle dottrine degli Israeliti ogni vestigio di sovrannaturale e di divino ; quella storia e quelle dottrine riduce spesso a favola; la Scrittura crede libro non pure non ispirato, ma ancora pieno di fole e di leggende; e di grado in grado, come era da prevedersi, giunge alle più sfacciate asserzioni di materialismo, di evoluzionismo, di ateismo ed alla negazione completa d'ogni Provvidenza divina. Di questi errori io non debbo far qui confutazione. Debbo solo notare che questo è lo spirito di ogni pagina dell'opera e che a questo risponde l'indefinita serie degli errori più smaccati e madornali, de'quali ho dato appena un piccolo saggio ${ }^{43}$.

Au centre de ce résumé, il est question d'une dégradation progressive et prévisible, d'étape en étape. Au commencement était la philologie rationaliste, cette méthode qui naturalise la Bible en excluant de l'histoire et de la doctrine d'Israël toute trace de surnaturel. Le texte saint est réduit à une simple fable, sans inspiration et pleine de fantaisies délirantes. Ce type de méthode a des conséquences doctrinales «prévisibles »: l'athéisme. Quelques années plus tard, le même Tripepi, dans des censures de Zola, montre la solidarité entre le naturalisme comme méthode littéraire et le naturalisme philosophique (négation du surnaturel, synonyme de matérialisme et d'athéisme). C'est le même lien de conséquence dont il parle ici. Le choix méthodologique d'une érudition excluant de ses analyses le facteur surnaturel conduit à une croyance matérialiste. Au-delà de la censure circonstanciée, le ton de Tripepi s'apparente à une leçon de morale ou un avertissement, peu de temps avant que n'éclate la crise moderniste.

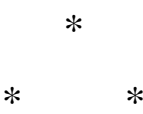

\footnotetext{
${ }^{42}$ Renan, Souvenirs d'enfance et de jeunesse, dans Euvres complètes, éd. Henriette Psichari, Paris, CalmannLévy, t. II, 1948, p. 851-852. À ce sujet, voir Keith Gore, L'Idée de progrès dans la pensée de Renan, Paris, Nizet, 1970, p. 156-159.

${ }^{43}$ Ibid., p. 18.
} 
L'ouverture des archives historiques du Saint-Office et de l'index a permis de prendre la mesure du regard romain sur l'œuvre de Renan. Jusqu'à une date récente, on la savait condamnée, on en devinait à grands traits les motifs. Renan suscite à Rome un intérêt constant et hors normes ; il est l'auteur le plus lu par le tribunal de l'Index, avec le plus de suivi, et le plus grand nombre de décrets de proscription pour le $\mathrm{XIX}^{\mathrm{e}}$ siècle. En dépit de cette attention exceptionnelle, il ne bénéficie pas de censures semblables à ses contemporains considérés comme appartenant au champ ecclésiastique : aucun texte, décret ou encyclique, ne définit les erreurs de Renan; et s'il suscite l'intérêt de la Curie, notamment de la nonciature à Paris et de la Secrétairerie d'État, il n'est pas même examiné par le Saint-Office, tribunal plus prestigieux que l'Index pour juger des menaces sur la doctrine catholique.

Les motifs de mise à l'Index sont essentiellement de deux ordres, philologique et doctrinal. Le choix méthodologique d'une érudition «rationaliste» pour étudier la Bible, c'est-à-dire ne prenant pas en considération l'idée d'intervention surnaturelle dans l'Histoire ni la tradition ecclésiastique interprétative des Écritures, conduit à une lecture naturalisante du texte sacré, qui lui récuse, quant à son origine, l'inspiration divine et, quant à son sens, un crédit en ce qui concerne les passages fabuleux. Du point de vue doctrinal, les rapporteurs de l'Index reprochent progressivement à l'œuvre de Renan son rationalisme philosophique, virant au panthéisme et à l'évolutionnisme, le dernier d'entre eux voyant même dans le matérialisme et l'évolutionnisme darwinien une sorte de conséquence de long terme des choix philologiques.

Jean-Baptiste Amadieu 
Annexe : Tableau archivistique des procédures en mises à l'Index de Renan

\begin{tabular}{|c|c|c|c|c|}
\hline \multirow{2}{*}{$\begin{array}{l}\text { Date du } \\
\text { décret }\end{array}$} & \multirow[t]{2}{*}{ Titre condamné } & \multicolumn{3}{|c|}{ Pièces des Protocolli des ACDF } \\
\hline & & année & folio & Type de doc \\
\hline \multirow[t]{4}{*}{$11 / 04 / 1859$} & \multirow[t]{4}{*}{ Le Livre de Job } & \multirow{4}{*}{$\begin{array}{l}1858- \\
61\end{array}$} & $\mathrm{~N}^{\circ} 34$ & Décret \\
\hline & & & abs & Relation CG \\
\hline & & & abs & $\begin{array}{l}\text { Invitation CG. Date CP : } \\
\text { 02/04/59 : } \\
\text { Duplicati delle Posizioni } \\
\text { (1855-1859) }\end{array}$ \\
\hline & & & $\mathrm{N}^{\circ} 35$ & Votum (8 p.) de Smith \\
\hline \multirow[t]{5}{*}{$07 / 07 / 1859$} & $\begin{array}{l}\text { Étude d'histoire } \\
\text { religieuse }\end{array}$ & \multirow[t]{5}{*}{$\begin{array}{l}1858- \\
61\end{array}$} & $\mathrm{~N}^{\circ} 40$ & Décret \\
\hline & \multirow{2}{*}{$\begin{array}{l}\text { De l'origine } d u \\
\text { langage }\end{array}$} & & abs & Relation CG \\
\hline & & & $\mathrm{N}^{\circ} 39$ & $\begin{array}{l}\text { Invitation CG. Date CP : } \\
\mathbf{0 2 / 0 7 / 5 9}\end{array}$ \\
\hline & $\begin{array}{l}\text { Histoire générale et } \\
\text { systèmer comparé } \\
\text { des } \quad \text { langues } \\
\text { sémitiques }\end{array}$ & & $\mathrm{N}^{\circ} 48$ & $\begin{array}{l}\text { Votum }(13 \text { p. }) \text { de Vercellone } \\
\text { Sur : Étude d'histoire } \\
\text { religieuse; } \\
\text { De l'origine du langage; } \\
\text { Histoire générale et système } \\
\text { comparé des langues } \\
\text { sémitiques. }\end{array}$ \\
\hline & $\begin{array}{l}\text { Averroès } \quad \text { et } \\
\text { l'averrö̈sme }\end{array}$ & & $\mathrm{N}^{\circ} 47$ & $\begin{array}{l}\text { Votum }(11 \mathrm{p} .) \text { de Semenenko } \\
\text { Sur : Averroès et l'averrö̈sme }\end{array}$ \\
\hline \multirow[t]{4}{*}{$10 / 09 / 1860$} & \multirow{4}{*}{$\begin{array}{l}\text { Le Cantique des } \\
\text { cantiques }\end{array}$} & \multirow{4}{*}{$\begin{array}{l}1858- \\
61\end{array}$} & $\mathrm{~N}^{\circ} 78$ & Décret \\
\hline & & & abs & Relation CG \\
\hline & & & $\mathrm{N}^{\circ} 77$ & $\begin{array}{l}\text { Invitation CG. Date CP : } \\
\mathbf{0 3 / 0 9 / 6 0}\end{array}$ \\
\hline & & & $\mathrm{N}^{\circ} 79$ & Votum (6 p.) de Smith \\
\hline \multirow[t]{4}{*}{$24 / 08 / 1863$} & \multirow[t]{4}{*}{ Vie de Jésus } & \multirow{4}{*}{$\begin{array}{l}1862- \\
64\end{array}$} & abs & Décret : Duplicati dei decreti \\
\hline & & & abs & Relation CG \\
\hline & & & Dossier & $\begin{array}{l}\text { Invitation CG. Date CP : } \\
\mathbf{1 8 / 0 8 / 6 3}\end{array}$ \\
\hline & & & & $\begin{array}{l}\text { Votum de Modena? } \\
\text { Rien sur Renan : voir le } \\
\text { diaire. }\end{array}$ \\
\hline \multirow[t]{4}{*}{$11 / 06 / 1866$} & \multirow[t]{4}{*}{ Les Apôtres } & \multirow{4}{*}{$\begin{array}{l}1865- \\
69\end{array}$} & $N^{\circ} 85$ & Décret \\
\hline & & & abs & Relation CG \\
\hline & & & $\mathrm{N}^{\circ} 73$ & $\begin{array}{l}\text { Invitation CG. Date CP : } \\
\mathbf{0 7 / 0 6 / 6 6}\end{array}$ \\
\hline & & & $\mathrm{N}^{\circ} 83$ & Votum (2 p.) de Vercellone \\
\hline \multirow[t]{3}{*}{$12 / 07 / 1869$} & $\begin{array}{l}\text { Questions } \\
\text { contemporaines }\end{array}$ & \multirow[t]{3}{*}{$\begin{array}{l}1865- \\
69\end{array}$} & $\mathrm{~N}^{\circ} 195$ & Décret : Duplicati dei decreti \\
\hline & \multirow[t]{2}{*}{ Saint Paul } & & abs & Relation CG \\
\hline & & & $\mathrm{N}^{\circ} 184$ & Invitation CG. Date CP : \\
\hline
\end{tabular}




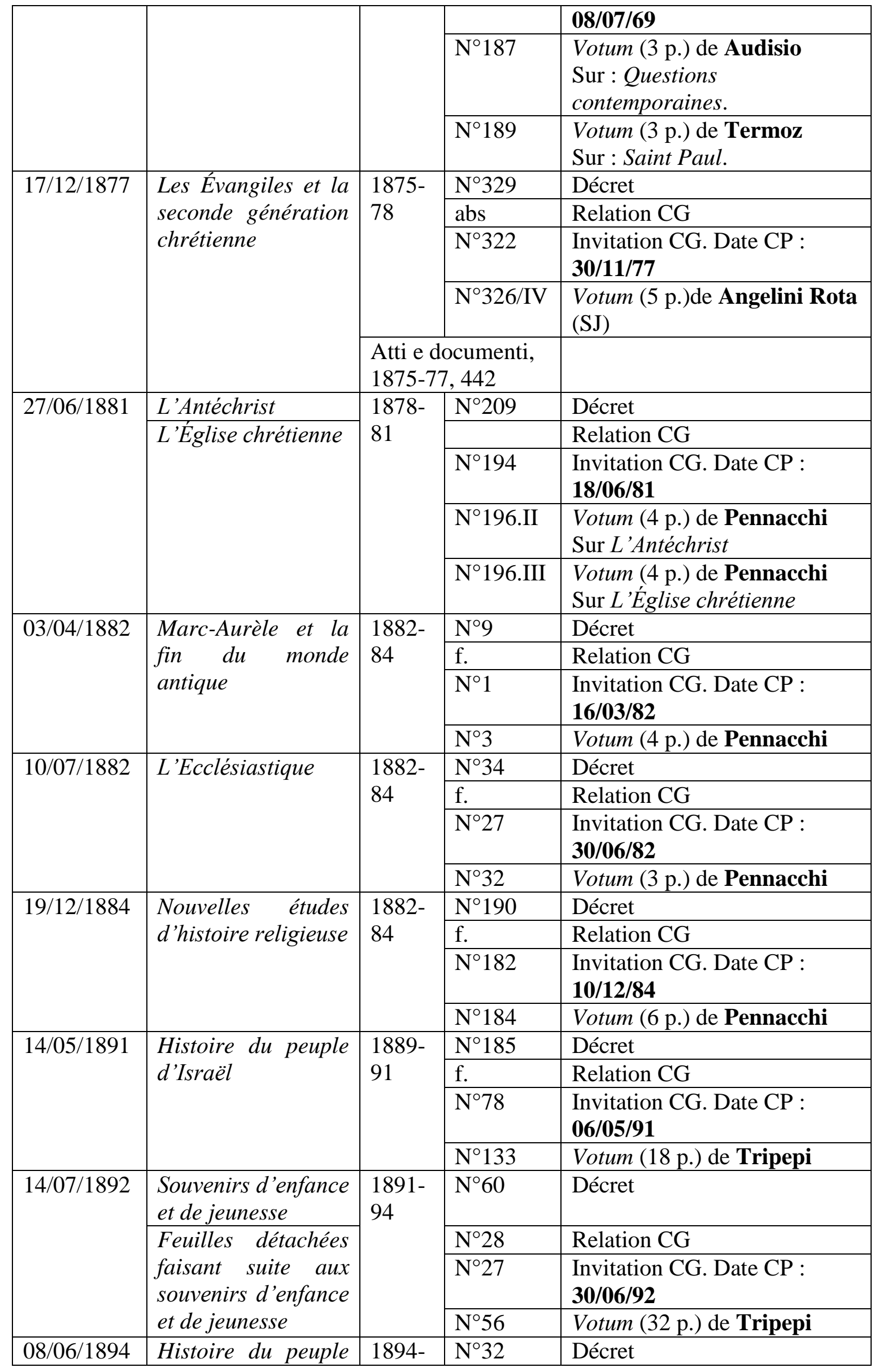




\begin{tabular}{|l|l|l|l|l|}
\hline \multirow{2}{*}{ d'Israël } & \multirow{2}{*}{96} & $\mathrm{~N}^{\circ} 30$ & Relation CG \\
\cline { 3 - 4 } & & $\mathrm{N}^{\circ} 18$ & $\begin{array}{l}\text { Invitation CG. Date CP : } \\
\mathbf{0 1 / 0 6 / 9 4}\end{array}$ \\
\cline { 3 - 4 } & & $\mathrm{N}^{\circ} 28$ & Votum $(43$ p.) de Tripepi \\
\hline
\end{tabular}

